\title{
Magnet tracking allows assessment of regional gastrointestinal transit times in children
}

This article was published in the following Dove Press journal:

Clinical and Experimental Gastroenterology

18 November 2013

Number of times this article has been viewed

Caroline Hedsund ${ }^{1,2}$

Iben Moeller Joensson ${ }^{2}$

Tine Gregersen'

Lotte Fynne'

Vincent Schlageter ${ }^{3}$

Klaus Krogh'

'Neurogastroenterology Unit, Department of Hepatology and Gastroenterology, ${ }^{2}$ Department of Pediatrics, Aarhus University Hospital, Aarhus, Denmark; ${ }^{3}$ Motilis Medica SA, Lausanne, Switzerland
Correspondence: Caroline Hedsund Neurogastroenterology Unit, Department of Hepatology and Gastroenterology V, Aarhus University Hospital, Nørrebrogade 44, DK-8000 Aarhus C, Denmark

Tel +4529849448

Fax +45 78462860

Email c_hedsund@hotmail.com
Background: Data on small intestinal transit time in healthy children are lacking, and normal values for gastric emptying and colonic transit time are sparse. Conventional methods, including radiopaque markers, scintigraphy, and PillCam ${ }^{\mathrm{TM}}$ involve radiation or require the child to swallow a large pill. The minimally invasive, radiation-free Motility Tracking System-1 (MTS-1) has been introduced for description of gastrointestinal motility in adults. The aim of the study was to evaluate the MTS-1 for assessment of gastrointestinal transit times and motility patterns in healthy children.

Methods: Twenty-one healthy children (nine girls), median age 10 (range 7-12) years were included. For evaluation with MTS-1, a small magnetic pill was ingested and tracked through the gastrointestinal tract by a matrix of 16 magnetic sensors placed behind a nonmagnetic bed. The children were investigated for 8 hours after swallowing the magnetic pill and again for 4 hours the following morning. After leaving the unit, each child came back after every bowel movement to determine if the pill had been expelled.

Results: Nineteen children could swallow the pill. Characteristic contraction patterns were identified for the stomach (three per minute), small intestine (9-11 per minute), and colon (4-5 per minute). Median total gastrointestinal transit time was 37.7 (range 9.5-95.8) hours, median gastric emptying time was 37 (range 2-142) minutes, median small intestinal transit time was 302 (range 164 to $>454$ ) minutes, and median colorectal transit time was 38.1 (range 5.6-90.0) hours.

Conclusion: MTS-1 allows minimally invasive evaluation of gastrointestinal motility in children. Use of the method is, however, restricted by the nonambulatory setup.

Keywords: gastrointestinal motility, gastrointestinal transit time, healthy children, motility tracking system

\section{Background}

Gastrointestinal symptoms are extremely common in children and adolescents. Among otherwise healthy school children, 3.5\%-27\% have dyspepsia, ${ }^{1,2} 6 \%-14 \%$ have irritable bowel syndrome, ${ }^{3}$ and up to $8 \%$ have functional constipation. ${ }^{4}$ In children with neurologic disorders, gastrointestinal dysfunction is even more common. Thus, $93 \%$ of children with cerebral palsy have some gastrointestinal disorder ${ }^{5}$ and $68 \%$ of children with myelomeningocele have abnormal defecation. ${ }^{6}$ Gastrointestinal disorders have severe consequences for daily life and quality of life in both the children affected and their families. ${ }^{7,8}$ Unfortunately, many children continue to have symptoms into adulthood. ${ }^{9,10}$

The most commonly used methods for evaluation of gastrointestinal or colorectal transit include scintigraphy and radiopaque markers. ${ }^{11}$ Gastrointestinal contractility 
can be evaluated by manometry, usually requiring endoscopy. Exposure to irradiation or discomfort is of special concern in children and restricts objective evaluation of gastrointestinal motility in daily clinical practice. Also, ethical considerations make normative data on bowel function in children extremely difficult to collect.

Early magnetic tracking studies with high resolution data on gastrointestinal transit have used multichannel superconducting quantum interference, with the drawbacks of requiring a shielded room and expensive equipment. ${ }^{12,13}$ The Motility Tracking System (MTS-1, Motilis Medica SA, Lausanne, Switzerland) is a new, minimally invasive, and radiation-free method for describing gastrointestinal transit times and motility. With MTS-1, a small magnetic pill is ingested and subsequently followed by external field sensors. The method has previously been validated in healthy adults ${ }^{14}$ and used in various patient groups. ${ }^{15-17}$

The aim of this study was to evaluate whether the MTS-1 is suitable for use in children and to describe basic contraction patterns and segmental transit times of the entire gastrointestinal tract in healthy children.

\section{Materials and methods Subjects}

Twenty-one healthy children (12 boys, median age 10 [range 7-12] years, median body mass index 16.7 [range 14.2-21.2] $\mathrm{kg} / \mathrm{m}^{2}$ ) were included after approval by the local scientific ethical committee (Project ID M-20100114) and informed oral and written consent by the children and their parents. All children were without symptoms of constipation or other bowel complaints, none had undergone gastrointestinal surgery, and none took medication affecting gastrointestinal function.

\section{Motility tracking system}

The MTS-1 allows minimally invasive assessment of gastric emptying time, small intestinal transit time, colorectal transit time, and total gastrointestinal transit time. Furthermore, region-specific contraction frequencies and movement patterns can be described.

A small magnetic pill $\left(6 \times 18 \mathrm{~mm}\right.$, density $\left.1.8 \mathrm{~g} / \mathrm{cm}^{3}\right)$ coated with a plastic capsule is swallowed (Figure 1). Afterwards, its movements are tracked by a detector plate of 16 magnetic field sensors $(4 \times 4)$ with a frequency of $10 \mathrm{~Hz}$ (Figure 1). Data from each sensor with an iterative algorithm are used to calculate the position and orientation of the magnetic pill. The umbilicus is used as a landmark to estimate placement of the detector plate in relation to the abdomen.

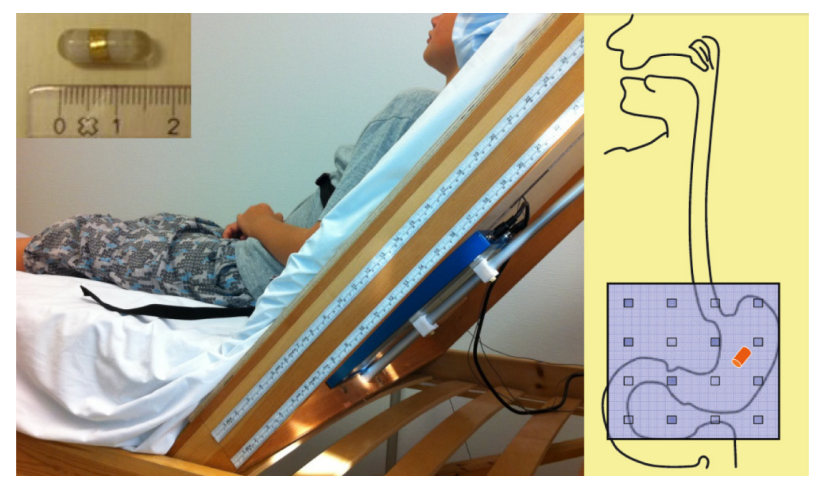

Figure I The blue sensor plate, placed behind the bed, tracks the movements of the magnetic pill. Data are transmitted via Bluetooth for computer analysis and display. The magnetic pill is shown above.

A vibration sensor is placed around the chest of the subject to identify respiratory movements and movement due to talking. Data are transferred through Bluetooth to a computer where they are visualized and analyzed in a customized software program (MTS record, Motilis Medica SA). Further technical details have been described previously. ${ }^{14}$

\section{Experimental protocol}

After 3 hours of fasting, the magnetic pill was ingested at $8 \mathrm{am}$. It was taken with half a glass of water, if possible, or else with a tablespoon of yoghurt or jam. During recordings, the child was sitting in a wooden bed with the head elevated to $45^{\circ}$. The detector plate was placed on the back of the bed, allowing a small table to be placed in front of the child so he/she could be occupied with, eg, drawing and playing. Standardized breaks of 5 minutes were held every hour, allowing the child to move around in the department. Recordings were obtained on 2 consecutive days. The firstday recordings were performed until 4 pm or stopped earlier if the magnetic pill reached the cecum.

Breakfast was served as the magnet passed from the stomach into the duodenum, and after a further 3 hours, lunch was provided. After fasting from midnight, the second day of recordings was resumed at $8 \mathrm{am}$. Breakfast was served at $10 \mathrm{am}$ and recordings were finished at $12 \mathrm{am}$, or earlier if the magnetic pill was passed with stool. All meals were standardized, and the energy levels of the meals were calculated according to the Nordic Nutrition Recommendations $2004^{18}$ and determined by the child's age, gender, and weight.

After recordings were stopped at noon on the second day, the child kept a stool diary. The child came in for an ambulatory visit after each defecation to determine whether the magnetic pill was still in the child and to conclude with which defecation the pill had passed. 


\section{Diaries}

The children and parents were instructed to fill out a stool diary from the first day of recordings until passing of the magnetic pill. Time of defecation and stool form were noted according to the Bristol Stool Scale. ${ }^{19}$ Time and type of meals were registered throughout the study.

\section{Data analysis}

MTS-1 recordings were reviewed and analyzed independently by two observers. Gastric emptying was defined as an abrupt shift from the highly characteristic contraction frequency of three per minute in the stomach to a fast, more irregular rhythm of eleven per minute in the duodenum accompanied by a characteristic movement in two dimensions as illustrated in Figure 2. Cessation of high-amplitude contractions of 9-10 per minute in the small intestine and shift to slow and low-amplitude contractions marked the ileocecal passage. Based on these data, gastric emptying and small intestinal transit time were calculated. Using the information from the stool diary and ambulatory visits to estimate the time of the defecation with which the magnetic pill left the body of the child, colorectal and total gastrointestinal or whole gut transit times were determined.

Velocity patterns for movement of the magnetic pill were calculated during the first 4 hours in the small intestine (on day 1) and in the colon for 2 hours during fasting and 2 hours postprandially (on day 2). Based on a previously performed analysis of velocity histograms identifying a trimodal distribution, movements were divided into fast ( $>15 \mathrm{~cm}$ per minute), slow ( $1.5-15 \mathrm{~cm}$ per minute), and very slow $\left(<1.5 \mathrm{~cm}\right.$ per minute).$^{20}$ Transit times for boys and girls were compared using the Mann-Whitney $U$ test. $P<0.05$ was considered to be statistically significant.

\section{Results}

Two boys (aged 8 years and 10 years) were unable to swallow the magnetic pill. In the remaining 19 , the investigation was well tolerated without any side effects. The number of stools per day was $0.7-1.7$ (median 1) and the value on the Bristol Stool Scale was 3-4.5 (median 3.5).

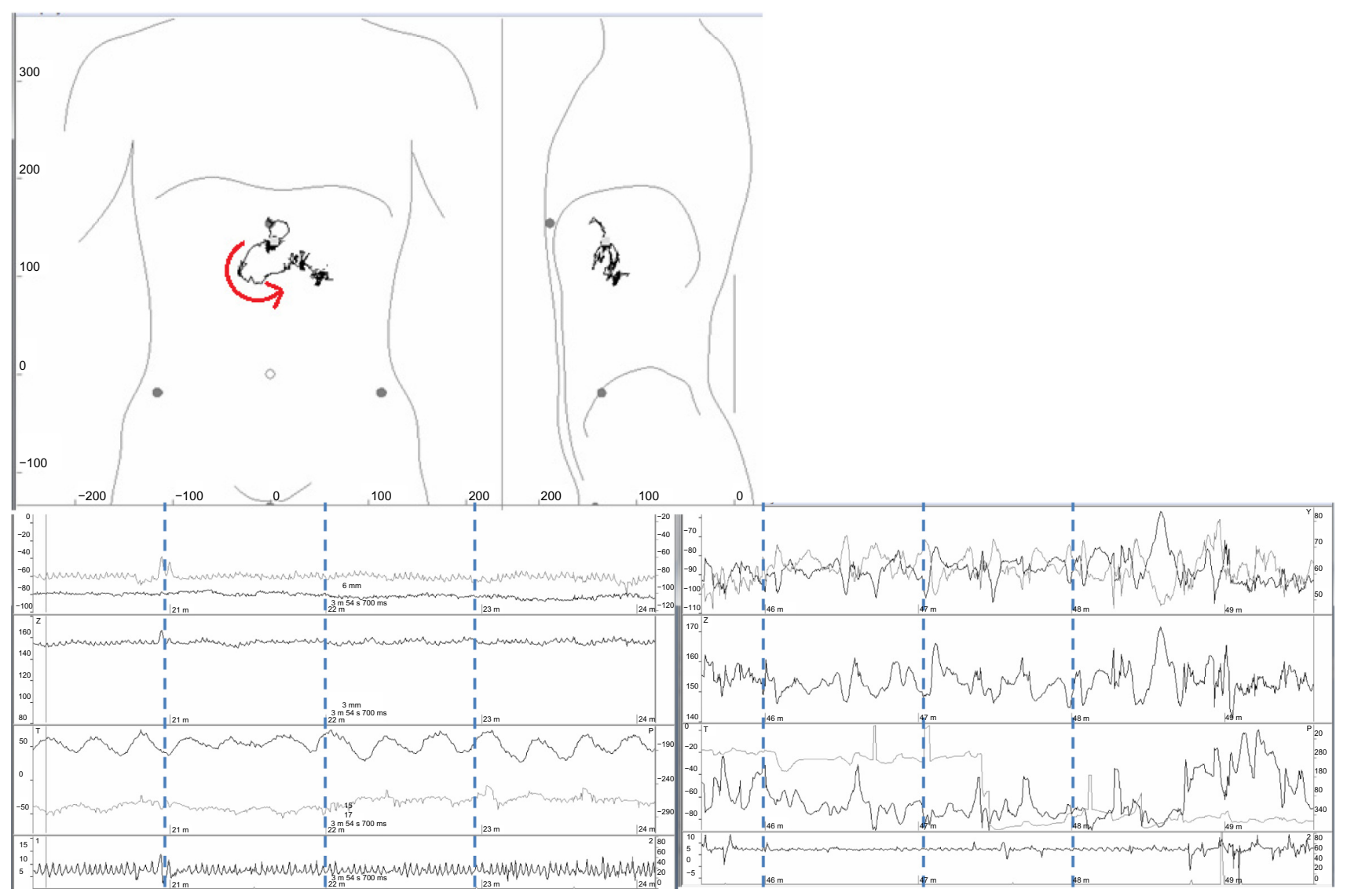

Figure 2 Gastric emptying illustrated by the MTS-I. In the two-dimensional plot (top), the rapid passage of the duodenal arch is illustrated by the arrow indicating the direction of movement. The corresponding change in contraction frequency from three per minute in the stomach (bottom left) to $9-11$ in the duodenum (bottom right) is shown below. The lines of the diaphragm, from above, shows movements in three directions $(x, y, z)$, two inclination angles $(\varphi, \theta)$ and movement registered by the breath sensor. The right and left panels show the amplitude. Time between the stippled lines is one minute. 


\section{Gastrointestinal transit times}

Total gastrointestinal and segmental transit times for each individual are shown in Table 1. Values for total gastrointestinal transit time, gastric emptying time, small intestinal transit time, and colorectal transit time are shown in Table 2. There were no differences in transit times between boys and girls. In five children, the magnetic pill did not pass into the cecum during the 8-hour protocol, making exact computation of their small intestinal and colorectal transit times impossible. In one of these children, recordings were stopped prematurely due to misjudgment of the location of the magnetic pill by the observer. The other four children had an orocecal transit time longer than the 8-hour protocol.

\section{Gastrointestinal contraction patterns}

The basic frequency of gastric and small intestinal contractions was easily identifiable, with three contractions per minute in the stomach and 9-11 per minute in the small intestine (Figure 2). The frequency of contractions declined gradually as the magnetic pill progressed more distally in the small intestine. The velocity of the magnetic pill during its passage through the small intestine was highly irregular (Figure 3). Thus, the magnetic pill covered $55 \%$ of the distance in the small intestine during fast movements, accounting for only $3 \%$ of the time. The velocity of the magnetic pill was 1.23 (range $0.74-2.41$ ) $\mathrm{cm}$ per minute during the first 2 hours after pyloric passage, but decreased as the pill advanced through the small intestine.
Basic contractions of the colon had a frequency of approximately five per minute. They were of very low amplitude and could only be identified occasionally. Mass movements, defined as migrations, moving the magnetic pill more than $10 \mathrm{~cm}$ in 10 seconds, were observed in four children. During mass movements, the magnetic pill travelled a distance of $10-51 \mathrm{~cm}$ in $23-76$ seconds. In three children, mass movements appeared 11-26 minutes after the standardized meal and in the fourth child was observed 67 minutes before breakfast was given. An example of colonic mass movement is shown in Figure 4.

\section{Discussion}

The present study shows that the MTS-1 can be used for description of gastrointestinal motility in children aged 7-12 years. It has previously been shown in adults that MTS-1 allows identification of the characteristic basic contraction frequencies of three per minute in the stomach, 9-11 per minute in the small intestine, and 5-6 per minute in the colon. ${ }^{14,15}$ Further, it has been shown that the combination of changes in contraction frequency and characteristic movements of the magnetic pill allows valid estimates of pyloric and ileocecal passage. ${ }^{14}$ In the present study, the contraction frequencies previously described in adults were clearly visible in healthy children. This allowed minimally invasive estimation of gastric emptying and small intestinal transit. Total gastrointestinal transit time and colorectal transit time

Table I Gastrointestinal and segmental transit times for healthy children

\begin{tabular}{|c|c|c|c|c|c|}
\hline Subject & $\begin{array}{l}\text { Age, } \\
\text { years }\end{array}$ & $\begin{array}{l}\text { Total gastrointestinal } \\
\text { transit time (hours) }\end{array}$ & $\begin{array}{l}\text { Gastric emptying } \\
\text { time (minutes) }\end{array}$ & $\begin{array}{l}\text { Small intestinal transit } \\
\text { time (minutes) }\end{array}$ & $\begin{array}{l}\text { Colorectal transit } \\
\text { time (hours) }\end{array}$ \\
\hline I & 12 & 54.7 & 31 & 366 & 48.1 \\
\hline 2 & 10 & 59.0 & 142 & $>331$ & \\
\hline 3 & 10 & 81.4 & 66 & 380 & 73.9 \\
\hline 4 & 10 & 95.8 & 34 & 317 & 90.0 \\
\hline 5 & 9 & 46.4 & 11 & 226 & 42.4 \\
\hline 6 & 11 & 29.0 & 37 & 290 & 23.6 \\
\hline 7 & 10 & 27.3 & 13 & 164 & 24.4 \\
\hline 8 & 10 & 9.5 & 66 & 166 & 5.6 \\
\hline 9 & 10 & 35.0 & 49 & $>428$ & \\
\hline 10 & 10 & 27.0 & 63 & $>276$ & \\
\hline 11 & 10 & 48.7 & 2 & 372 & 42.4 \\
\hline 12 & 8 & 49.3 & 42 & 397 & 42.0 \\
\hline 13 & 12 & 30.4 & 111 & 181 & 25.5 \\
\hline 14 & 9 & 56.6 & 21 & $>454$ & \\
\hline 15 & 10 & 30.1 & 16 & 204 & 26.4 \\
\hline 16 & 7 & 37.6 & 37 & 165 & 34.2 \\
\hline 17 & 12 & 37.7 & 37 & $>427$ & \\
\hline 18 & 12 & 25.2 & 108 & 314 & 18.2 \\
\hline 19 & 11 & 80.1 & 72 & 328 & 73.4 \\
\hline
\end{tabular}

Note: In five children, exact values for small intestinal and colorectal transit times are unavailable because ileocecal passage did not occur during the 8-hour protocol on the first day. 
Table 2 Gastrointestinal transit times in healthy children

\begin{tabular}{lllll}
\hline & All children & Boys & Girls & P-value \\
\hline Total gastrointestinal transit time (hours) & $37.7(9.5-95.8)$ & $40.7(9.5-81.4)$ & $37.7(27.3-95.8)$ & 0.44 \\
Gastric emptying time (minutes) & $37(2-142)$ & $56(2-142)$ & $37(13-111)$ & 0.54 \\
Small intestinal transit time (minutes)* & $302(164-397)$ & $314(116-380)$ & $204(164-397)$ & 0.38 \\
Colorectal transit time (hours)* & $38.1(5.6-90.0)$ & $42.4(5.6-73.0)$ & $34.2(24.4-73.3)$ & 0.71 \\
\hline
\end{tabular}

Notes: Values are given as the median (range). *In five children, values for small intestinal and colorectal transit times were unavailable as ileocecal passage did not occur during the 8-hour protocol on the first day. This difference in numbers explains why median colorectal transit time can be slightly longer than the total gastrointestinal transit time.

could be calculated by controlling for the presence of the magnetic pill after each defecation.

In addition to providing data on total and segmental gastrointestinal transit times, the MTS-1 allowed description of various movement patterns. Small intestinal transit time and motility was only studied in the postprandial state, but the passage was highly irregular, as most of the distance was covered in only $3 \%$ of time. Colonic mass movements were clearly visible, and the distance covered during each could be estimated.

Existing methods for description of gastrointestinal transit times in children mainly include radiopaque markers and scintigraphy. Radiopaque markers are commonly used in research and clinical practice. ${ }^{21}$ The protocols used are easy to follow and allow ambulant estimation of gastrointestinal transit and segmental colorectal transit times. Drawbacks with the use of radiopaque markers are exposure to irradiation and lack of information about gastric emptying and small intestinal transit time. Scintigraphy, in contrast, provides precise information about gastric emptying, small intestinal transit time, and segmental colorectal transit. However, the method is expensive, not readily available, and exposes the child to irradiation. For these reasons, the use of scintigraphy for evaluation of gastrointestinal motility in children has been very limited. ${ }^{22}$

Data on small intestinal transit time are extremely sparse. Fallingborg et al have studied regional transit times of the bowel in healthy children using a $\mathrm{pH}$ capsule,,$^{23}$ and orocecal transit time has been investigated using the lactulose hydrogen breath test. ${ }^{24} \mathrm{~A}$ few studies in healthy children have investigated colonic transit times using radiopaque markers. ${ }^{22,25,26}$ In some of these, the children had to have an X-ray taken for other reasons, so the term "healthy children" may not be fully correct. Our population of healthy children was recruited through advertisement and not through an outpatient clinic or after having other procedures done in the hospital.

In accordance with previous MTS-1studies in adults, intersubject variation among children was very large. Variation in gastric emptying time was particularly large. This was to some extent caused by the design of our study. In most subjects, an object the size of the magnetic pill will pass the pylorus during phase 3 of the migrating motor complex. ${ }^{27}$ If the magnetic pill was taken with food, it would likely stay

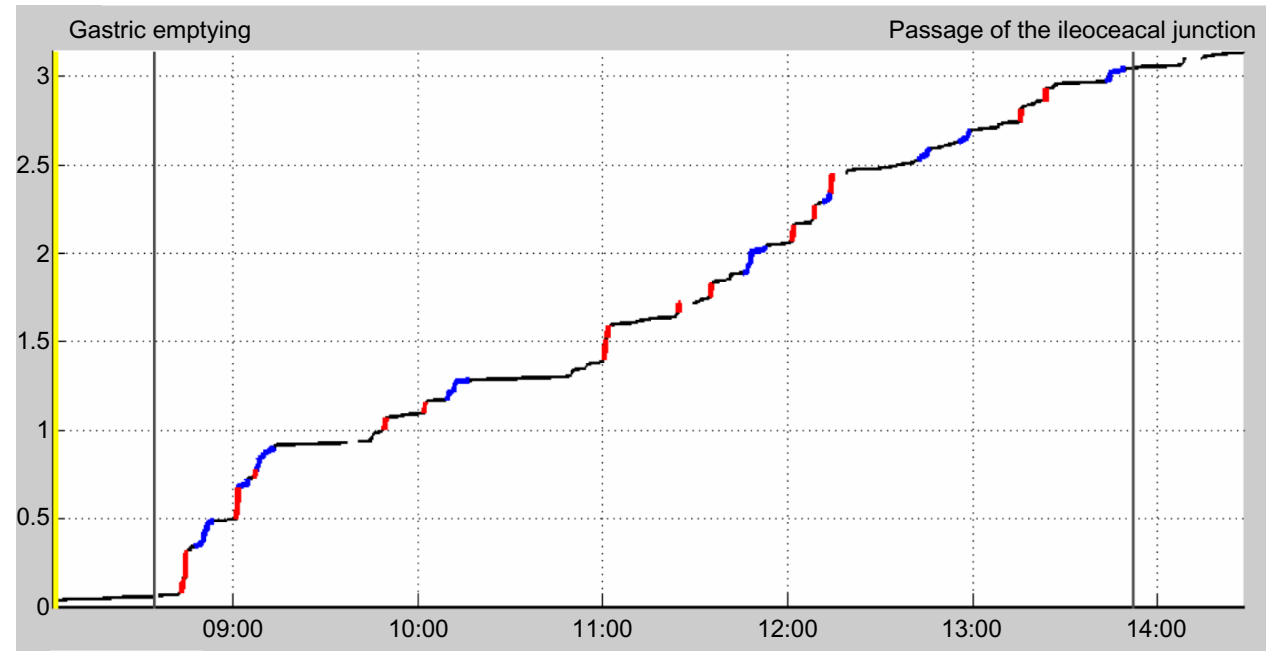

Figure 3 The time (hours) versus distance (meters) curve for progression of the magnetic pill through the small intestine. Immobile periods are shown in black, slow movements in blue, and fast movements in red. 


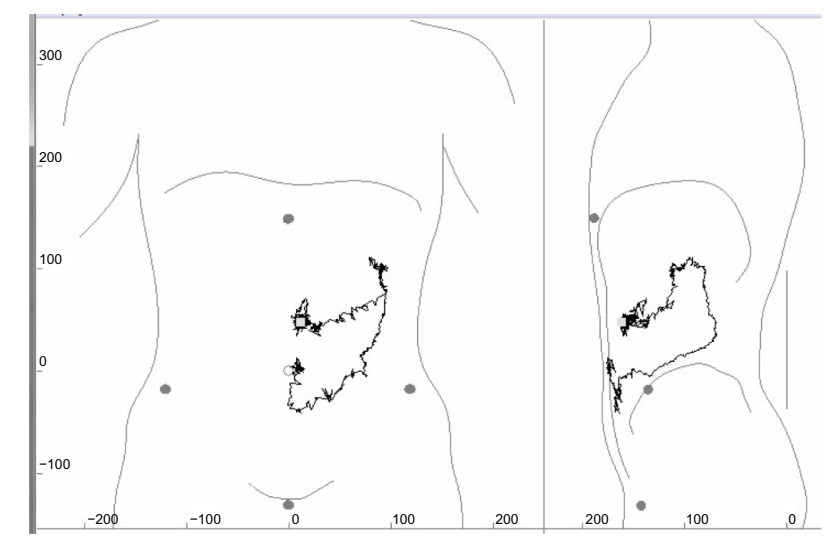

Figure 4 Example of a colonic mass movement. The magnetic pill moved $51 \mathrm{~cm}$ from the transverse colon to the sigmoid colon in 76 seconds.

in the stomach for hours until the occurrence of the fasting motility pattern. This would be inconsistent with the 8-hour time limit of our protocol. Therefore, the magnetic pill was ingested in the fasting state and the first meal was served just after pyloric passage. However, five children did not succeed in ingesting the magnetic pill with water, but did so with yogurt or jam. This is a potential bias, but estimated as small. The design of this study allowed standardized recordings from the small intestine within a reasonable time. Gastric emptying, in contrast, would vary because it depends on the time from intake of the pill to the next phase 3 of the migrating motor complex. ${ }^{27}$ The density and size of the foreign object in the bowel is essential when measuring physiologic transit times. Taking into account the smaller anatomic properties in children, it might have a greater influence than when studying adults. This may lead to delay in gastric emptying and prolonged transit times.

The large variation in total gastrointestinal and colorectal transit times is in accordance with previous studies using radiopaque markers in children and adults. ${ }^{22}$ We find that this variation must reflect normal physiology. In previous studies, various definitions of the upper limit for normal total gastrointestinal transit time have been used. Arhan et al defined prolonged colonic transit time as longer than 62 hours $^{26}$ and Wagener et al defined the cutoff value as 84 hours. ${ }^{25}$ In a review by Southwell et al, the upper limit for normal total gastrointestinal transit time was set at 32 (range 25-40) hours..$^{22}$ Applying the frequently used upper limit of 62 hours, three of the 19 children investigated with the MTS-1 in the present study had a prolonged transit time. An explanation of slow transit times measured in the present study might be that the children were kept still during recordings. Future development of the technique towards an ambulatory system would eliminate this potential bias. Also, in accordance with previous studies using other methods, we found no differences in either total or regional transit times when comparing girls and boys. ${ }^{21}$

There are limitations with our study and with the use of the MTS-1 in general. The most important is that the system is stationary, so confines the subject studied to sitting in a bed. This is far from the daily activities of a healthy child and limits the study protocol. For ethical reasons, we followed the magnetic pill for 8 hours after oral intake. Further, we had to allow the children to move for 5 minutes each hour. The time limit meant that we missed ileocecal passage in a proportion of the children, but since breaks did not exceed 5 minutes, this did not have a great influence on the overall results. The same problem has been encountered in studies of adult patients, but the limitations were to some extent avoided by calculating the velocity of the magnetic pill during the first hours after pyloric passage. This approach is not ideal, but has allowed us to identify abnormalities in patients with spinal cord injury, ${ }^{16}$ systemic sclerosis, ${ }^{15}$ cystic fibrosis, ${ }^{28}$ and carcinoid syndrome. ${ }^{17}$ Compared with gastric emptying and small intestinal transit times, colorectal transit time is usually much longer. This restricts the usefulness of a nonambulatory system like the MTS-1 for providing a detailed description of colorectal motility. However, we did show that the MTS-1 can identify and quantify colonic mass movements in children.

The ambulatory, second-generation system, MTS-2 (Motilis Medica SA) is under development, and pilot data from healthy adults have been presented in abstract form. ${ }^{29}$ In adults, the MTS-2 allowed continuous and ambulatory description of gastrointestinal motility for up to 3 days. As shown in the present study, magnet tracking for description of gastrointestinal transit patterns is possible, and future application of the novel ambulatory system could avoid most of the limitations encountered with the present system. Another ambulatory system, the Wireless Motility Capsule or SmartPill $^{\mathrm{TM}}$ (Smart-Pill Corporation, Buffalo, NY, USA) is registered for clinical use in adults. ${ }^{30}$ It allows safe and easy estimation of gastric emptying, small intestinal transit time, and total colorectal transit time, but does not allow assessment of segmental colonic transit time or quantification of colonic mass movements. To the best of our knowledge, there are no published data on the use of the Wireless Motility Capsule in children. The size of the magnetic pill is of special concern in children. It is possible to reduce the size of permanent magnetic pills. However, the magnetic capsule developed for ambulatory use is larger than those used in the present study. 


\section{Conclusion}

We have shown that the MTS- 1 can provide a detailed description of gastrointestinal transit and motility in healthy children. The method is safe, and most children can easily swallow the magnet. However, use of the MTS-1 is restricted by the stationary nature of the system. Ongoing development of an ambulatory system could hold great promise for future studies designed to show whether characteristic abnormalities can be found in children with various gastrointestinal disorders. The ambulatory magnet tracking system, as introduced for adults, has great potential for investigation of gastrointestinal motility in children.

\section{Acknowledgment}

The study was supported by the Karen Elise Jensen Foundation and the Riisfort Foundation.

\section{Disclosure}

VS is co-owner of Motilis Medica SA. The other authors have no conflicts of interest in this work.

\section{References}

1. De Giacomo C, Valdambrini V, Lizzoli F, et al. A population-based survey on gastrointestinal tract symptoms and Helicobacter pylori infection in children and adolescents. Helicobacter. 2002;7(6): 356-363.

2. Hyams JS, Davis P, Sylvester FA, Zeiter DK, Justinich CJ, Lerer T. Dyspepsia in children and adolescents: a prospective study. J Pediatr Gastroenterol Nutr. 2000;30(4):413-418.

3. Hyams JS, Burke G, Davis PM, Rzepski B, Andrulonis PA. Abdominal pain and irritable bowel syndrome in adolescents: a community-based study. J Pediatr. 1996;129(2):220-226.

4. Loening-Baucke V. Constipation in early childhood: patient characteristics, treatment, and longterm follow up. Gut. 1993;34(10): 1400-1404.

5. Del Guidice E, Staiano A, Capano G, et al. Gastrointestinal manifestations in children with cerebral palsy. Brain Dev. 1999;21(5):307-311.

6. Krogh K, Lie HR, Bilenberg N, Laurberg S. Bowel function in Danish children with myelomeningocele. APMIS Suppl. 2003;(109):81-85.

7. Clarke MC, Chow CS, Chase JW, Gibb S, Hutson JM, Southwell BR. Quality of life in children with slow transit constipation. J Pediatr Surg. 2008;43(2):320-324

8. Kaugars AS, Silverman A, Kinservik M, et al. Families' perspectives on the effect of constipation and fecal incontinence on quality of life. J Pediatr Gastroenterol Nutr. 2010;51(6):747-752.

9. Bongers ME, Benninga MA, Maurice-Stam H, Grootenhuis MA. Health-related quality of life in young adults with symptoms of constipation continuing from childhood into adulthood. Health Qual Life Outcomes. 2009;7:20

10. Khan S, Campo J, Bridge JA, Chiappetta LC, Wald A, Di Lorenzo C. Long-term outcome of functional childhood constipation. Dig Dis Sci. 2007;52(1):64-69.
11. Sutcliffe JR, King S, Hutson JM, Southwell B. What is new in radiology and pathology of motility disorders in children? Semin Pediatr Surg. 2010;19(2):81-85.

12. Weitschies W, Kotitz R, Cordini D, Trahms L. High-resolution monitoring of the gastrointestinal transit of a magnetically marked capsule. J Pharm Sci. 1997;86(11):1218-1222.

13. Weitschies W, Karaus M, Cordini D, Trahms L, Breitkreutz J, Semmler W. Magnetic marker monitoring of disintegrating capsules. Eur J Pharm Sci. 2001;13(4):411-416.

14. Worsoe J, Fynne L, Gregersen T, et al. Gastric transit and small intestinal transit time and motility assessed by a magnet tracking system. $B M C$ Gastroenterol. 2011;11(1):145.

15. Fynne L, Worsoe J, Gregersen T, Schlageter V, Laurberg S, Krogh K. Gastrointestinal transit in patients with systemic sclerosis. Scand J Gastroenterol. 2011;46(10):1187-1193.

16. Fynne L, Worsoe J, Gregersen T, Schlageter V, Laurberg S, Krogh K. Gastric and small intestinal dysfunction in spinal cord injury patients. Acta Neurol Scand. 2012;125(2):123-128.

17. Gregersen T, Gronbaek H, Worsoe J, Schlageter V, Laurberg S, Krogh K. Effects of Sandostatin LAR on gastrointestinal motility in patients with neuroendocrine tumors. Scand J Gastroenterol. 2011;46(7-8):895-902.

18. Becker W, Lynhe N, Pedersen AN, et al. Nordic Nutrition Recommendations 2004 - integrating nutrition and physical activity. Scandinavian Journal of Nutrition. 2004;48(4):178-187.

19. Heaton KW, Radvan J, Cripps H, Mountford RA, Braddon FE, Hughes AO. Defecation frequency and timing, and stool form in the general population: a prospective study. Gut. 1992;33(6):818-824.

20. Hiroz P, Schlageter V, Givel JC, Kucera P. Colonic movements in healthy subjects as monitored by a magnet tracking system. Neurogastroenterol Motil. 2009;21(8):838. e57.

21. National Institute for Health and Clinical Excellence. Guidance: constipation in children and young people: diagnosis and management of idiopathic childhood constipation in primary and secondary care. 2010. Available from: http://www.nice.org.uk/cg99. Accessed September 1, 2013.

22. Southwell BR, Clarke MC, Sutcliffe J, Hutson JM. Colonic transit studies: normal values for adults and children with comparison of radiological and scintigraphic methods. Pediatr Surg Int. 2009;25(7):559-572.

23. Fallingborg J, Christensen LA, Ingeman-Nielsen M, et al. Measurement of gastrointestinal $\mathrm{pH}$ and regional transit times in normal children. $J$ Pediatr Gastroenterol Nutr. 1990;11(2):211-214.

24. Murphy MS, Nelson R, Eastham EJ. Measurement of small intestinal transit time in children. Acta Paediatr Scand. 1988;77(6):802-806.

25. Wagener S, Shankar KR, Turnock RR, Lamont GL, Baillie CT. Colonic transit time - what is normal? J Pediatr Surg. 2004;39(2):166-169.

26. Arhan P, Devroede G, Jehannin B, et al. Segmental colonic transit time. Dis Colon Rectum. 1981;24(8):625-629.

27. Cassilly D, Kantor S, Knight LC, et al. Gastric emptying of a nondigestible solid: assessment with simultaneous SmartPill $\mathrm{pH}$ and pressure capsule, antroduodenal manometry, gastric emptying scintigraphy. Neurogastroenterol Motil. 2008;20(4):311-319.

28. Hedsund C, Gregersen T, Joensson IM, Olesen HV, Krogh K. Gastrointestinal transit times and motility in patients with cystic fibrosis Scand J Gastroenterol. 2012;47(8-9):920-926.

29. Haase A, Gregersen T, Fynne L, et al. Gastrointestinal contraction patterns and segmental transit times evaluated with ambulatory capsule tracking. Neurogastroenterol Motil. 2012;24 Suppl 2:187.

30. Maqbool S, Parkman HP, Friedenberg FK. Wireless capsule motility: comparison of the SmartPill GI monitoring system with scintigraphy for measuring whole gut transit. Dig Dis Sci. 2009;54(10):2167-2174. 


\section{Publish your work in this journal}

Clinical and Experimental Gastroenterology is an international, peerreviewed, open access journal, publishing all aspects of gastroenterology in the clinic and laboratory, including: Pathology, pathophysiology of gastrointestinal disease; Investigation and treatment of gastointestinal disease; Pharmacology of drugs used in the alimentary tract;
Immunology/genetics/genomics related to gastrointestinal disease. This journal is indexed on CAS. The manuscript management system is completely online and includes a very quick and fair peer-review system. Visit http://www.dovepress.com/testimonials.php to read real quotes from published authors.

Submit your manuscript here: http://www.dovepress.com/clinical-and-experimental-gastroenterology-journal 